\title{
A Two-parameter Generalized Skew-Cauchy Distribution
}

\author{
Wahab Bahrami ${ }^{\dagger, *}$, Hojat Rangin ${ }^{\ddagger}$ and Kauomars Rangin ${ }^{\star}$ \\ $\dagger$ Shahid Bahonar University of Kerman \\ ¥ Tarbiat Moalem University of Tehran \\ * Razi University of Kermanshah
}

\begin{abstract}
In this paper, we discuss a new generalization of univariate skewCauchy distribution with two parameters, we denoted this by $\operatorname{GSC}\left(\lambda_{1}, \lambda_{2}\right)$, that it has more flexible than the skew-Cauchy distribution (denoted by SC $(\lambda)$ ), introduced by Behboodian et al. (2006). Furthermore, we establish some useful properties of this distribution and by two numerical example, show that $\operatorname{GSC}\left(\lambda_{1}, \lambda_{2}\right)$ can fits the data better than $\operatorname{SC}(\lambda)$.
\end{abstract}

Keywords. Generalized skew-Cauchy; generalized skew-normal; skew-Cauchy and skew-normal distributions.

\section{Introduction}

Azzalini $(1985,1986)$ introduced the standard skew-normal distribution as a generalization of the normal distribution. A random variable $Z_{\lambda}$ has a standard skew-normal distribution with parameter $\lambda \in \mathbb{R}$, denoted by $\operatorname{SN}(\lambda)$, if its pdf is

$$
f(z ; \lambda)=2 \phi(z) \Phi(\lambda z) \quad z \in \mathbb{R},
$$

where $\phi(\cdot)$ and $\Phi(\cdot)$ are the standard normal pdf and cdf, respectively. This distribution has been studied and generalized by some researchers. Jamalizadeh et al. (2008) discussed a new class of skew-normal distribution with two parameters. A random variable $Z_{\lambda_{1}, \lambda_{2}}$ has a two-parameter generalized skew-normal distribution with parameters $\lambda_{1}, \lambda_{2} \in \mathbb{R}$, denoted by

* Corresponding author 
$\operatorname{GSN}\left(\lambda_{1}, \lambda_{2}\right)$, if its pdf is

$$
f\left(z, \lambda_{1}, \lambda_{2}\right)=\frac{2 \pi}{\cos ^{-1}\left(-\frac{\lambda_{1} \lambda_{2}}{\sqrt{1+\lambda_{1}^{2}} \sqrt{1+\lambda_{2}^{2}}}\right)} \phi(z) \Phi\left(\lambda_{1} z\right) \Phi\left(\lambda_{2} z\right) \quad z \in \mathbb{R},
$$

also, they established some simple and useful properties of this distribution.

If $X \sim \mathrm{N}(0,1)$ be independent of $Z_{\lambda}$, it is easy to show that $\frac{Z_{\lambda}}{X} \sim C(0,1)$ for $\lambda \in \mathbb{R}$. However $W_{\lambda}=\frac{Z_{\lambda}}{|X|}$ when $\lambda \neq 0$ is not $C(0,1)$. Behboodian et al. (2006) refer to it as a skew-Cauchy distribution with parameter $\lambda \in \mathbb{R}$ and denoted it by $W_{\lambda} \sim \mathrm{SC}(\lambda)$. They derived the density of $W_{\lambda}$ as follows

$$
f(w ; \lambda)=\frac{1}{\pi\left(1+w^{2}\right)}\left(1+\frac{\lambda w}{\sqrt{1+\left(1+\lambda^{2}\right) w^{2}}}\right) \quad w \in \mathbb{R},
$$

and discussed some simple and important characteristics of this distribution. Let $X \sim \mathrm{N}(0,1)$ be independent of $Z_{\lambda_{1}, \lambda_{2}}$. In this paper, we consider the distribution of $W_{\lambda_{1}, \lambda_{2}}=\frac{Z_{\lambda_{1}, \lambda_{2}}}{|X|}$, refer to it as a two-parameter generalized skew-Cauchy distribution and denote this by $\operatorname{GSC}\left(\lambda_{1}, \lambda_{2}\right)$.

This paper is organized as follows. In the next section we derive the density of $W_{\lambda_{1}, \lambda_{2}}$ and present some simple properties of this distribution. In Section 3 we discuss about the moments of $\operatorname{GSC}\left(\lambda_{1}, \lambda_{2}\right)$. Some important properties of $\operatorname{GSC}\left(\lambda_{1}, \lambda_{2}\right)$ are given in Section 4, and in Section 5, two numerical examples to compare GSC and SC are provided.

\section{Two-parameter Generalized Skew-Cauchy Dis- tribution}

In this section, we derive the density of $W_{\lambda_{1}, \lambda_{2}}$ and establish some simple properties of this distribution.

Definition 1. A random variable $W_{\lambda_{1}, \lambda_{2}}$ has a two-parameter generalized skew-Cauchy distribution with parameters $\lambda_{1}, \lambda_{2} \in \mathbb{R}$, if $W_{\lambda_{1}, \lambda_{2}} \stackrel{d}{=} \frac{Z_{\lambda_{1}, \lambda_{2}}}{|X|}$, where $Z_{\lambda_{1}, \lambda_{2}} \sim \operatorname{GSN}\left(\lambda_{1}, \lambda_{2}\right)$ and $X \sim \mathrm{N}(0,1)$ are independent.

To obtain the density of $W_{\lambda_{1}, \lambda_{2}}$, let $g\left(w ; \lambda_{1}, \lambda_{2}\right)$ and $G\left(w ; \lambda_{1}, \lambda_{2}\right)$ denote the pdf and cdf of $W_{\lambda_{1}, \lambda_{2}}$, respectively. Then

$$
G\left(w ; \lambda_{1}, \lambda_{2}\right)=P\left(Z_{\lambda_{1}, \lambda_{2}} \leqslant w|X|\right)=E\left\{\Phi\left(w|X| ; \lambda_{1}, \lambda_{2}\right)\right\},
$$


where $\Phi\left(\cdot ; \lambda_{1}, \lambda_{2}\right)$ is the cdf of $Z_{\lambda_{1}, \lambda_{2}} \sim \operatorname{GSN}\left(\lambda_{1}, \lambda_{2}\right)$. We have

$$
G\left(w ; \lambda_{1}, \lambda_{2}\right)=2 \int_{0}^{\infty} \Phi\left(w x ; \lambda_{1}, \lambda_{2}\right) \phi(x) d x,
$$

which, by differentiation, we obtain

$$
g\left(w ; \lambda_{1}, \lambda_{2}\right)=\frac{2}{\cos ^{-1}\left(-\frac{\lambda_{1} \lambda_{2}}{\sqrt{1+\lambda_{1}^{2}} \sqrt{1+\lambda_{2}^{2}}}\right)} \int_{0}^{\infty} x e^{-\frac{1}{2} x^{2}\left(1+w^{2}\right)} \Phi\left(\lambda_{1} w x\right) \Phi\left(\lambda_{2} w x\right) d x .
$$

If

$$
\begin{gathered}
g_{1}\left(w ; \lambda_{1}, \lambda_{2}\right)=\frac{\lambda_{1} w}{\sqrt{1+\left(1+\lambda_{1}^{2}\right) w^{2}}}\left\{\frac{1}{4}+\frac{1}{2 \pi} \tan ^{-1}\left(\frac{\lambda_{2} w}{\sqrt{1+\left(1+\lambda_{1}^{2}\right) w^{2}}}\right)\right\}, \\
g_{2}\left(w ; \lambda_{1}, \lambda_{2}\right)=\frac{\lambda_{2} w}{\sqrt{1+\left(1+\lambda_{2}^{2}\right) w^{2}}}\left\{\frac{1}{4}+\frac{1}{2 \pi} \tan ^{-1}\left(\frac{\lambda_{1} w}{\sqrt{1+\left(1+\lambda_{2}^{2}\right) w^{2}}}\right)\right\}
\end{gathered}
$$

and $a=\frac{2 \pi}{\cos ^{-1}\left(-\frac{\lambda_{1} \lambda_{2}}{\sqrt{1+\lambda_{1}^{2}} \sqrt{1+\lambda_{2}^{2}}}\right)}$, then by integration by parts, we obtain the density of $W_{\lambda_{1}, \lambda_{2}}$ as

$$
g\left(w ; \lambda_{1}, \lambda_{2}\right)=\frac{a}{\pi\left(1+w^{2}\right)}\left\{\frac{1}{4}+g_{1}\left(w ; \lambda_{1}, \lambda_{2}\right)+g_{2}\left(w ; \lambda_{1}, \lambda_{2}\right)\right\} .
$$

If $\lambda_{1}=\lambda_{2}=\lambda$ and $b=\frac{\pi}{\tan ^{-1}\left(\sqrt{1+2 \lambda^{2}}\right)}$ then the above density reduce to

$$
\begin{aligned}
g(w ; \lambda)= & \frac{b}{\pi\left(1+w^{2}\right)}\left[\frac{1}{4}+\frac{\lambda w}{\sqrt{1+\left(1+\lambda^{2}\right) w^{2}}}\right. \\
& \left.\times\left\{\frac{1}{2}+\frac{1}{\pi} \tan ^{-1}\left(\frac{\lambda w}{\sqrt{1+\left(1+\lambda^{2}\right) w^{2}}}\right)\right\}\right] .
\end{aligned}
$$

If the pdf of a variable is (1), we denote this by $\operatorname{GSC}\left(\lambda_{1}, \lambda_{2}\right)$, and if the pdf of a variable is (2), then we denote this by $\operatorname{GSC}(\lambda)$. Figure 1 illustrates several of the possible shapes obtained from $\operatorname{GSC}\left(\lambda_{1}, \lambda_{2}\right)$ under various choices of $\left(\lambda_{1}, \lambda_{2}\right)$. Some simple properties of $\operatorname{GSC}\left(\lambda_{1}, \lambda_{2}\right)$ is presented as follows.

Theorem 1. 1. GSC $(0,0)=C(0,1)$. 
2. $\operatorname{GSC}\left(\lambda_{1}, 0\right)=\operatorname{SC}\left(\lambda_{1}\right)$ and $\operatorname{GSC}\left(0, \lambda_{2}\right)=\operatorname{SC}\left(\lambda_{2}\right)$.

3. $\operatorname{GSC}\left(\lambda_{1}, \lambda_{2}\right)=\operatorname{GSC}\left(\lambda_{2}, \lambda_{1}\right)$.

4. $W_{\lambda_{1}, \lambda_{2}} \sim \operatorname{GSC}\left(\lambda_{1}, \lambda_{2}\right) \Leftrightarrow-W_{\lambda_{1}, \lambda_{2}} \stackrel{d}{=} W_{-\lambda_{1},-\lambda_{2}} \sim \operatorname{GSC}\left(-\lambda_{1},-\lambda_{2}\right)$.

5. If $X, X_{1}, X_{2}, X_{3} \stackrel{i i d}{\sim} \mathrm{N}(0,1)$ and $X_{1: 3} \leqslant X_{2: 3} \leqslant X_{3: 3}$ be the corresponding order statistics, then

$$
\frac{X_{1: 3}}{|X|} \sim \operatorname{GSC}(-1,-1), \quad \frac{X_{2: 3}}{|X|} \sim \operatorname{GSC}(1,-1), \quad \frac{X_{3: 3}}{|X|} \sim \operatorname{GSC}(1,1) .
$$

Proof. The parts 1, 2, 3 and 4 are easily obtained from Definition 1 and the density of $W_{\lambda_{1}, \lambda_{2}}$. For part 5, we know that

$$
X_{1: 3} \sim \operatorname{GSN}(-1,-1), \quad X_{2: 3} \sim \operatorname{GSN}(1,-1), \quad X_{3: 3} \sim \operatorname{GSN}(1,1),
$$

(see Jamalizadeh et al., 2008), thus by Definition 1 the proof is completed.

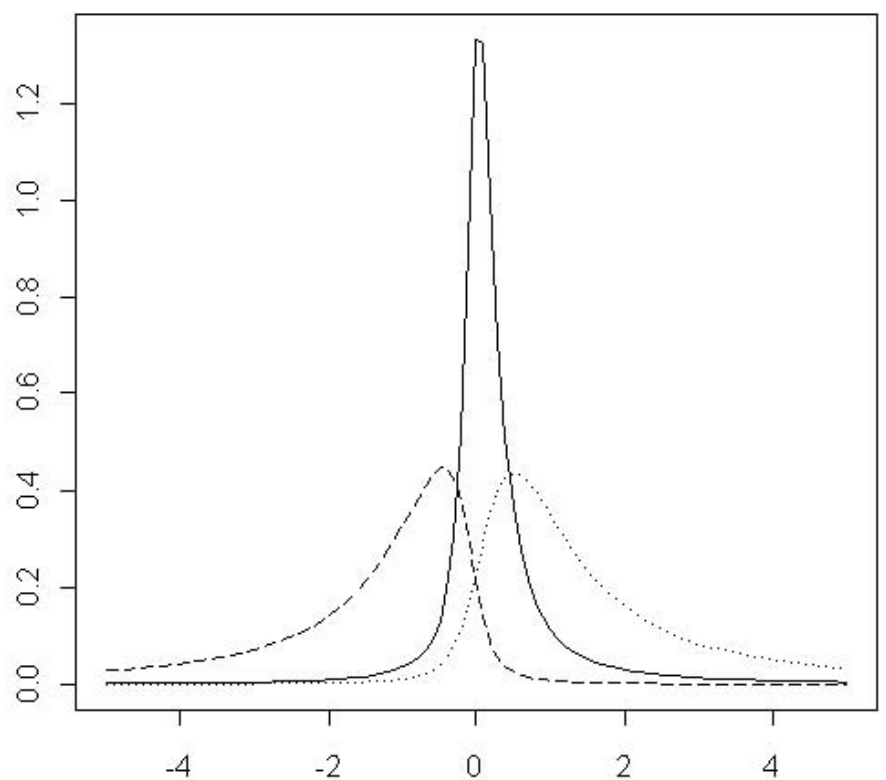

Figure 1. Example of the $\operatorname{GSC}\left(\lambda_{1}, \lambda_{2}\right)$ density for $\left(\lambda_{1}, \lambda_{2}\right)=(-3,5)$ (solid line), $\left(\lambda_{1}, \lambda_{2}\right)=$ $(1,2)$ (dotted line), $\left(\lambda_{1}, \lambda_{2}\right)=(-1,-3)$ (dashed line). 


\section{Moments}

In this section we discuss about the moments of $\operatorname{GSC}\left(\lambda_{1}, \lambda_{2}\right)$. We show that the odd moments of two-parameter generalized skew-Cauchy distribution are divergent. Suppose that $W_{\lambda_{1}, \lambda_{2}} \sim \operatorname{GSC}\left(\lambda_{1}, \lambda_{2}\right)$. By the Definition 1 , we have

$$
E\left(W_{\lambda_{1}, \lambda_{2}}^{m}\right)=E\left(\frac{Z_{\lambda_{1}, \lambda_{2}}^{m}}{|X|^{m}}\right)
$$

where $m=1,2, \ldots$. Since $Z_{\lambda_{1}, \lambda_{2}}$ and $X$ are independent and $Y=|X|^{2} \sim \chi_{1}^{2}$, then

$$
E\left(W_{\lambda_{1}, \lambda_{2}}^{m}\right)=E\left(Z_{\lambda_{1}, \lambda_{2}}^{m}\right) E\left(Y^{-\frac{m}{2}}\right)
$$

Let $a=\frac{2 \pi}{\cos ^{-1}\left(-\frac{\lambda_{1} \lambda_{2}}{\sqrt{1+\lambda_{1}^{2}} \sqrt{1+\lambda_{2}^{2}}}\right)}$. Now, we calculate $E\left(Z_{\lambda_{1}, \lambda_{2}}^{m}\right)$ and $E\left(Y^{-\frac{m}{2}}\right)$.

$$
\begin{aligned}
E\left(Z_{\lambda_{1}, \lambda_{2}}^{m}\right)= & a \int_{-\infty}^{+\infty} z^{m-1} z \phi(z) \Phi\left(\lambda_{1} z\right) \Phi\left(\lambda_{2} z\right) d z \\
= & a(m-1) \int_{-\infty}^{+\infty} z^{m-2} \phi(z) \Phi\left(\lambda_{1} z\right) \Phi\left(\lambda_{2} z\right) d z \\
& +\frac{a \lambda_{1}}{\sqrt{2 \pi}} \int_{-\infty}^{+\infty} z^{m-1} \phi\left(\sqrt{1+\lambda_{1}^{2}} z\right) \Phi\left(\lambda_{2} z\right) d z \\
& +\frac{a \lambda_{2}}{\sqrt{2 \pi}} \int_{-\infty}^{+\infty} z^{m-1} \phi\left(\sqrt{1+\lambda_{2}^{2}} z\right) \Phi\left(\lambda_{1} z\right) d z \\
= & a(m-1) E\left(Z_{\lambda_{1}, \lambda_{2}}^{m-2}+\frac{a \lambda_{1}\left(1+\lambda_{1}^{2}\right)^{-\frac{m}{2}}}{2 \sqrt{2 \pi}}\right. \\
& \times E\left(Y_{1}^{m-1}\right)+\frac{a \lambda_{2}\left(1+\lambda_{2}^{2}\right)^{-\frac{m}{2}}}{2 \sqrt{2 \pi}} E\left(Y_{2}^{m-1}\right),
\end{aligned}
$$

where $Y_{1} \sim \operatorname{SN}\left(\frac{\lambda_{1}}{\sqrt{1+\lambda_{1}^{2}}}\right)$ and $Y_{2} \sim \operatorname{SN}\left(\frac{\lambda_{2}}{\sqrt{1+\lambda_{2}^{2}}}\right)$.

The moments generating function of $S \sim \mathrm{SN}(\lambda)$ is

$$
M_{S}(t)=2 e^{\frac{t^{2}}{2}} \Phi\left(\frac{\lambda t}{\sqrt{1+\lambda^{2}}}\right)
$$

and also, we have

$$
E\left(Y^{-\frac{m}{2}}\right)=\int_{0}^{+\infty} \frac{1}{\sqrt{2 \pi}} y^{-\frac{m}{2}} e^{-\frac{y}{2}} y^{-\frac{1}{2}} d y=\frac{\Gamma\left(\frac{1-m}{2}\right)}{\sqrt{\pi} 2^{\frac{m}{2}}} .
$$


Since the gamma function is divergent for all nonpositive integers, thus $E\left(Y^{-\frac{m}{2}}\right)$ is divergent for $m=1,3, \ldots$. Therefore, if $m=2,4, \ldots$, then

$$
\begin{aligned}
E\left(W_{\lambda_{1}, \lambda_{2}}^{m}\right)= & \frac{a \Gamma\left(\frac{1-m}{2}\right)}{\sqrt{\pi} 2^{\frac{m}{2}}}\left\{(m-1) E\left(Z_{\lambda_{1}, \lambda_{2}}^{m-2}\right)\right. \\
& \left.+\frac{\lambda_{1}\left(1+\lambda_{1}^{2}\right)^{-\frac{m}{2}}}{2 \sqrt{2 \pi}} E\left(Y_{1}^{m-1}\right)+\frac{\lambda_{2}\left(1+\lambda_{2}^{2}\right)^{-\frac{m}{2}}}{2 \sqrt{2 \pi}} E\left(Y_{2}^{m-1}\right)\right\} .
\end{aligned}
$$

For a special case

$$
\begin{aligned}
E\left(W_{\lambda_{1}, \lambda_{2}}^{2}\right) & =\frac{a \Gamma\left(-\frac{1}{2}\right)}{2 \sqrt{\pi}}\left\{1+\frac{\lambda_{1}}{2 \sqrt{2 \pi}\left(1+\lambda_{1}^{2}\right)} E\left(Y_{1}\right)+\frac{\lambda_{2}}{2 \sqrt{2 \pi}\left(1+\lambda_{2}^{2}\right)} E\left(Y_{2}\right)\right\} \\
& =\frac{a \Gamma\left(-\frac{1}{2}\right)}{2 \sqrt{\pi}}\left\{1+\frac{\lambda_{1}^{2}}{2 \pi\left(1+\lambda_{1}^{2}\right) \sqrt{1+2 \lambda_{1}^{2}}}+\frac{\lambda_{2}^{2}}{2 \pi\left(1+\lambda_{2}^{2}\right) \sqrt{1+2 \lambda_{2}^{2}}}\right\} .
\end{aligned}
$$

The value of gamma function for some special cases that may has been used to calculating the moments as follows.

$$
\begin{aligned}
& \Gamma(-0.5)=-3.544908 \\
& \Gamma(-1.5)=2.363272 \\
& \Gamma(-2.5)=-0.9453087 \\
& \Gamma(-3.5)=0.2700882
\end{aligned}
$$

\section{Some Important Properties}

Theorem 2. Suppose that $X, U_{1}, U_{2}, U_{3} \stackrel{\text { iid }}{\sim} \mathrm{N}(0,1)$ and also, $Y_{1}=\frac{U_{1}}{|X|}$, $Y_{2}=\frac{U_{2}}{|X|}$ and $Y_{3}=\frac{U_{3}}{|X|}$. Then

$$
W_{\lambda_{1}, \lambda_{2}} \stackrel{d}{=} Y_{1} \mid\left(Y_{2}<\lambda_{1} Y_{1}, Y_{3}<\lambda_{2} Y_{1}\right) \sim \operatorname{GSC}\left(\lambda_{1}, \lambda_{2}\right) .
$$

Proof. It is clearly that

$$
W_{\lambda_{1}, \lambda_{2}} \stackrel{d}{=} \frac{U_{1}}{|X|} \mid\left(U_{2}<\lambda_{1} U_{1}, U_{3}<\lambda_{2} U_{1}\right) .
$$

Let $U \stackrel{d}{=} U_{1} \mid\left(U_{2}<\lambda_{1} U_{1}, U_{3}<\lambda_{2} U_{1}\right)$. We know that

$$
P\left(U_{2}<\lambda_{1} U_{1}, U_{3}<\lambda_{2} U_{1}\right)=\frac{\cos ^{-1}\left(-\frac{\lambda_{1} \lambda_{2}}{\sqrt{1+\lambda_{1}^{2}} \sqrt{1+\lambda_{2}^{2}}}\right)}{2 \pi},
$$


(see Jamalizadeh et al., 2008), and also

$$
\begin{aligned}
f_{U}(u) & =\frac{P\left(U_{2}<\lambda_{1} U_{1}, U_{3}<\lambda_{2} U_{1} \mid U_{1}=u\right) \phi(u)}{P\left(U_{2}<\lambda_{1} U_{1}, U_{3}<\lambda_{2} U_{1}\right)} \\
& =\frac{2 \pi}{\cos ^{-1}\left(-\frac{\lambda_{1} \lambda_{2}}{\sqrt{1+\lambda_{1}^{2}} \sqrt{1+\lambda_{2}^{2}}}\right)} \phi(u) \Phi\left(\lambda_{1} u\right) \Phi\left(\lambda_{2} u\right),
\end{aligned}
$$

then $U \sim \operatorname{GSN}\left(\lambda_{1}, \lambda_{2}\right)$ and therefore, by Definition 1 the proof is completed.

Corollary 1. Suppose that $X, U_{1}, U_{2}, U_{3} \stackrel{i i d}{\sim} \mathrm{N}(0,1)$ and also, $Y_{1}=\frac{U_{1}}{|X|}$, $Y_{2}=\frac{U_{2}}{|X|}$ and $Y_{3}=\frac{U_{3}}{|X|}$. Then

$$
Y_{1} \mid\left(Y_{2}<\lambda Y_{1}, Y_{3}<\lambda Y_{1}\right) \sim \operatorname{GSC}(\lambda) .
$$

Theorem 3. Suppose that $\left(U_{1}, U_{2}, U_{3}\right) \sim \mathrm{N}_{3}\left(\mathbf{0},\left(\begin{array}{ccc}1 & \rho_{12} & \rho_{13} \\ \rho_{12} & 1 & \rho_{23} \\ \rho_{13} & \rho_{23} & 1\end{array}\right)\right)$, with $\rho_{23}=\rho_{12} \rho_{13}$, and also $X \sim \mathrm{N}(0,1)$ be independent of $\left(U_{1}, U_{2}, U_{3}\right)$. Then

$$
W_{\lambda_{1}, \lambda_{2}} \stackrel{d}{=} \frac{U_{1}}{|X|} \mid\left(\min \left(U_{2}, U_{3}\right)>0\right) \sim \operatorname{GSC}\left(\lambda_{1}, \lambda_{2}\right),
$$

where $\lambda_{1}=\frac{\rho_{12}}{\sqrt{1-\rho_{12}^{2}}}$ and $\lambda_{2}=\frac{\rho_{13}}{\sqrt{1-\rho_{13}^{2}}}$.

Proof. Since $U_{1} \mid\left(\min \left(U_{2}, U_{3}\right)>0\right) \sim \operatorname{GSN}\left(\lambda_{1}, \lambda_{2}\right)$ (see Jamalizadeh et al., 2008), then by Definition 1 the proof is completed.

We need to the next definition and lemma to present the next theorem.

Definition 2. We say that $\mathbf{V}=\left(V_{1}, V_{2}, V_{3}\right)$ has a standard trivariate Cauchy distribution if its pdf is

$$
f(\mathbf{v} ; \Sigma)=\frac{1}{\pi^{2}|\Sigma|^{\frac{1}{2}}\left(1+\mathbf{v} \Sigma^{-1} \mathbf{v}\right)^{2}},
$$


where $\mathbf{v}^{\circ}=\left(v_{1}, v_{2}, v_{3}\right)$ and $\Sigma=\left(\begin{array}{ccc}1 & \rho_{12} & \rho_{13} \\ \rho_{12} & 1 & \rho_{23} \\ \rho_{13} & \rho_{23} & 1\end{array}\right)$, (see Fang et al., 1990).

We denote this distribution by $\mathbf{V} \sim C_{3}(\mathbf{0}, \Sigma)$. It can be shown that for $i=1,2,3$, we have $V_{i} \sim C(0,1)$.

Lemma 1. Suppose that $\left(U_{1}, U_{2}, U_{3}\right) \sim \mathrm{N}_{3}\left(\mathbf{0},\left(\begin{array}{ccc}1 & \rho_{12} & \rho_{13} \\ \rho_{12} & 1 & \rho_{23} \\ \rho_{13} & \rho_{23} & 1\end{array}\right)\right)$ and $X \sim \mathrm{N}(0,1)$ are independent. Then

$$
\left(\frac{U_{1}}{|X|}, \frac{U_{2}}{|X|}, \frac{U_{3}}{|X|}\right) \sim C_{3}(\mathbf{0}, \Sigma) .
$$

Proof. Suppose that $\mathbf{U}=\left(U_{1}, U_{2}, U_{3}\right) \sim \mathrm{N}_{3}(\mathbf{0}, \Sigma)$ and $\mathbf{V}=\left(V_{1}, V_{2}, V_{3}\right) \stackrel{d}{=}$ $\left(\frac{U_{1}}{|X|}, \frac{U_{2}}{|X|}, \frac{U_{3}}{|X|}\right)$, then

$$
\begin{aligned}
F_{\mathbf{V}}(\mathbf{v}) & =P(\mathbf{V} \leqslant \mathbf{v})=P\left(\frac{U_{1}}{|X|} \leqslant v_{1}, \frac{U_{2}}{|X|} \leqslant v_{2}, \frac{U_{3}}{|X|} \leqslant v_{3}\right) \\
& =P(\mathbf{U} \leqslant \mathbf{v}|X|)=E\left\{\Phi_{3}(\mathbf{v}|X| ; \Sigma)\right\} \\
& =2 \int_{0}^{\infty} \Phi_{3}(\mathbf{v} x ; \Sigma) \phi(x) d x,
\end{aligned}
$$

where $\Phi_{3}(\cdot ; \Sigma)$ is the cdf of $\mathrm{N}_{3}(\mathbf{0}, \Sigma)$. Upon differentiating this expression of $F_{\mathbf{V}}(\mathbf{v})$, we obtain

$$
f_{\mathbf{V}}(\mathbf{v})=\frac{\partial}{\partial v_{1} \partial v_{2} \partial v_{3}} F_{\mathbf{V}}(\mathbf{v})=2 \int_{0}^{\infty} x^{3} \frac{e^{-\frac{1}{2} x^{2}\left(\mathbf{v} \Sigma^{-1} \mathbf{v}\right)}}{(2 \pi)^{\frac{3}{2}}|\Sigma|^{\frac{1}{2}}} \cdot \frac{e^{-\frac{1}{2} x^{2}}}{\sqrt{2 \pi}} d x
$$

then, by integration by parts, we have

$$
\begin{aligned}
f_{\mathbf{V}}(\mathbf{v}) & =\frac{2}{(2 \pi)^{2}|\Sigma|^{\frac{1}{2}}}\left(-\frac{x^{2} e^{-\frac{1}{2} x^{2}\left(1+\mathbf{v} \Sigma^{-1} \mathbf{v}\right)}}{1+\mathbf{v} \Sigma^{-1} \mathbf{v}}-\frac{2 e^{-\frac{1}{2} x^{2}\left(1+\mathbf{v} \Sigma^{-1} \mathbf{v}\right)}}{\left(1+\mathbf{v} \Sigma^{-1} \mathbf{v}\right)^{2}}\right)_{0}^{\infty} \\
& =\frac{1}{\pi^{2}|\Sigma|^{\frac{1}{2}}\left(1+\mathbf{v} \Sigma^{-1} \mathbf{v}\right)^{2}} .
\end{aligned}
$$


Table 1. MLEs for the lifespan of rats (ad libitum diet) under GSC and SC models.

\begin{tabular}{cccc}
\hline \hline & SC $(\boldsymbol{\lambda})$ & $\operatorname{GSC}(\boldsymbol{\lambda})$ & $\operatorname{GSC}\left(\boldsymbol{\lambda}_{\mathbf{1}}, \boldsymbol{\lambda}_{\mathbf{2}}\right)$ \\
\hline$\widehat{\boldsymbol{\lambda}}$ & 0.2902438 & 0.09042141 & - \\
$\widehat{\boldsymbol{\lambda}}_{\mathbf{1}}$ & - & - & -1.656760 \\
$\widehat{\boldsymbol{\lambda}}_{\mathbf{2}}$ & - & - & 2.153499 \\
Log-likelihood & -137.655963 & -137.999645 & -119.832504 \\
\hline
\end{tabular}

Theorem 4. Suppose that $\left(V_{1}, V_{2}, V_{3}\right) \sim C_{3}(\mathbf{0}, \Sigma)$ and $\Sigma=\left(\begin{array}{ccc}1 & \rho_{12} & \rho_{13} \\ \rho_{12} & 1 & \rho_{23} \\ \rho_{13} & \rho_{23} & 1\end{array}\right)$ with $\rho_{23}=\rho_{12} \rho_{13}$. Then

$$
V_{1} \mid\left(\min \left(V_{2}, V_{3}\right)>0\right) \sim \operatorname{GSC}\left(\lambda_{1}, \lambda_{2}\right),
$$

where $\lambda_{1}=\frac{\rho_{12}}{\sqrt{1-\rho_{12}^{2}}}$ and $\lambda_{2}=\frac{\rho_{13}}{\sqrt{1-\rho_{13}^{2}}}$.

Proof. As in Lemma 1 , suppose that $\left(V_{1}, V_{2}, V_{3}\right) \stackrel{d}{=}\left(\frac{U_{1}}{|X|}, \frac{U_{2}}{|X|}, \frac{U_{3}}{|X|}\right)$, where $\left(U_{1}, U_{2}, U_{3}\right) \sim \mathrm{N}_{3}\left(\mathbf{0},\left(\begin{array}{ccc}1 & \rho_{12} & \rho_{13} \\ \rho_{12} & 1 & \rho_{23} \\ \rho_{13} & \rho_{23} & 1\end{array}\right)\right)$, with $\rho_{23}=\rho_{12} \rho_{13}$, and $X \sim$ $\mathrm{N}(0,1)$ are independent. Then the proof is completed by theorem 2 .

\section{Data Illustration}

In this section we consider two data sets to compare $\operatorname{SC}(\lambda)$ and $\operatorname{GSC}\left(\lambda_{1}, \lambda_{2}\right)$.

Example 1. This example considers the standardized data concerning the lifespan of rats that they were under an ad libitum diet (that is, "free eating"), given in Landau and Everitt (2003). We want to compare $\operatorname{SC}(\lambda)$ and $\operatorname{GSC}\left(\lambda_{1}, \lambda_{2}\right)$, by fitting them for these standardized data. We estimate parameters by numerically maximizing the likelihood function. The obtained numerical results are presented in Table 1. Based on log-likelihood, $\operatorname{GSC}\left(\widehat{\lambda}_{1}, \widehat{\lambda}_{2}\right)$ fits the data better than $\operatorname{SC}(\widehat{\lambda})$. Figure 2 illustrates the histogram of the data with the fitted densities.

Example 2. In this example, we consider the standardized roller data set, available for downloading at http://lib.stat.cmu.edu/jasadata/laslett and 
Table 2. MLEs for the roller data set under GSC and SC models

\begin{tabular}{cccc}
\hline \hline & $\operatorname{SC}(\boldsymbol{\lambda})$ & $\operatorname{GSC}(\boldsymbol{\lambda})$ & $\operatorname{GSC}\left(\boldsymbol{\lambda}_{\mathbf{1}}, \boldsymbol{\lambda}_{\mathbf{2}}\right)$ \\
\hline$\hat{\boldsymbol{\lambda}}$ & 0.1649845 & 0.0590169 & - \\
$\widehat{\boldsymbol{\lambda}}_{\mathbf{1}}$ & - & - & 1.580540 \\
$\widehat{\boldsymbol{\lambda}}_{\mathbf{2}}$ & - & - & -1.337220 \\
Log-likelihood & -1871.571888 & -1872.913647 & -1731.895581 \\
\hline
\end{tabular}

alternatively analyzed by Gomez et al. (2006). The data set consists of 1150 heights measured at 1 micron intervals along the drum of a roller (i.e. parallel to the axis of the roller). For this standardized data set the obtained numerical results are presented in Table 2. Based on log-likelihood, GSC $\left(\widehat{\lambda}_{1}, \widehat{\lambda}_{2}\right)$ fits the data better than $\operatorname{SC}(\hat{\lambda})$. This point is further illustrated in Figure 3, where a histogram of the data is plotted together with the fitted densities.

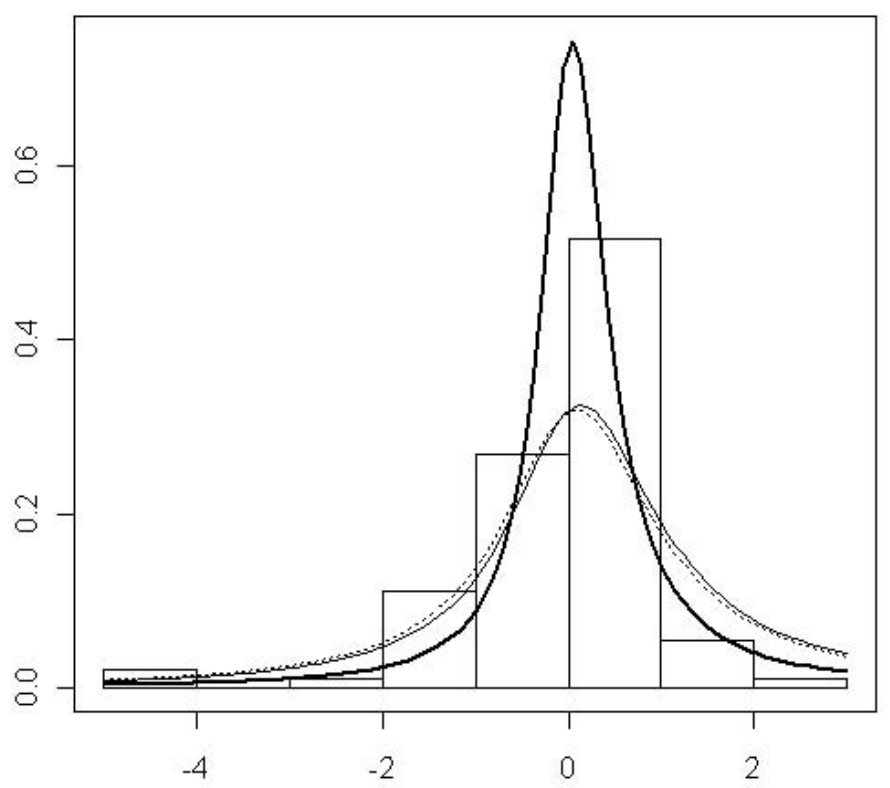

Figure 2. Histogram of the lifespan of rats (ad libitum diet). The lines represent distributions fitted using MLE: GSC $\left(\widehat{\lambda}_{1}, \widehat{\lambda}_{2}\right)$ (bold solid line), $\operatorname{GSC}(\widehat{\lambda})$ (dotted line), $\operatorname{SC}(\widehat{\lambda})$ (solid line). 


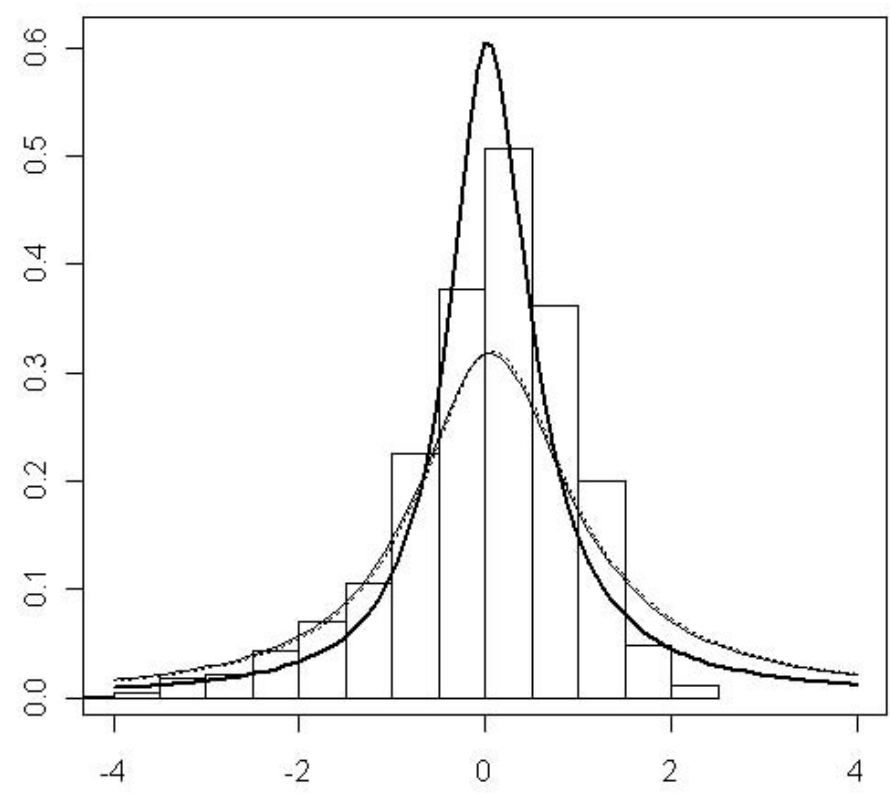

Figure 3. Histogram of the standardized roller data. The lines represent distributions fitted using MLE: $\operatorname{GSC}\left(\widehat{\lambda}_{1}, \widehat{\lambda}_{2}\right)$ (bold solid line), $\operatorname{GSC}(\widehat{\lambda})$ (dotted line), $\operatorname{SC}(\widehat{\lambda})$ (solid line).

\section{References}

Arnold, B.C. and Beaver, R.J. (2000). The skew-Cauchy distribution. Statistics and Probability Letters, 49, 285-290.

Azzalini, A. (1985). A class of distributions which includes the normal ones. Scandinavian Journal of Statistics, 12, 171-178.

Azzalini, A. (1986). Further results on a class of distributions which includes the normal ones. Statistica, 46, 199-208.

Bahrami, W., Agahi, H. and Rangin, H. (2010). A two-parameter Balakrishnan skew-normal distribution, J. Statist. Res. Iran, 6, 231-242.

Behboodian, J., Jamalizadeh, A. and Balakrishnan, N. (2006). A new class of skew-Cauchy distributions. Statistics and Probability Letters, 76, 1488-1493.

Fang, K.T., Kotz, S. and Ng, K.W. (1990). Symmetric Multivariate and Related Distributions. Chapman and Hall, London. 
Gomez, H.W., Salinas, H.S. and Bolfarine, H. (2006). Generalized skew-normal models: properties and inference. Statistics, 40, 495-505.

Henze, N.A. (1986). A probabilistic representation of the skew-normal distribution. Scandinavian Journal of Statistics, 13, 271-275.

Huang, W.J. and Chen, Y.H. (2007). Generalized skew-Cauchy distribution. Statistics and Probability Letters, 77, 1137-1147.

Jamalizadeh, A., Behboodian, J. and Balakrishnan, N. (2008). A two-parameter generalized skew-normal distribution. Statistics and Probability Letters, 78, 1722-1728.

Landau, S. and Everitt, B.S. (2003). A Handbook of Statistical Analyses using SPSS. Chapman and Hall, Boca Raton.

\section{Wahab Bahrami}

Department of Statistics,

Shahid Bahonar University of Kerman,

Kerman, Iran.

email:W.Bahrami@yahoo.com

\section{Hojat Rangin}

Department of Statistics,

Tarbiat Moalem University of Tehran,

Tehran, Iran.

\section{Kauomars Rangin}

Department of Statistics,

Razi University of Kermanshah,

Kermanshah, Iran. 